\title{
Detection of Posaconazole by Surface-Assisted Laser Desorption/Ionization Mass Spectrometry with Dispersive Liquid-Liquid Microextraction
}

\author{
Sheng-Yu Lin, Pin-Shiuan Chen, Sarah Y. Chang \\ Department of Chemistry, National Kaohsiung Normal University, Yanchao, Kaohsiung, Taiwan 824
}

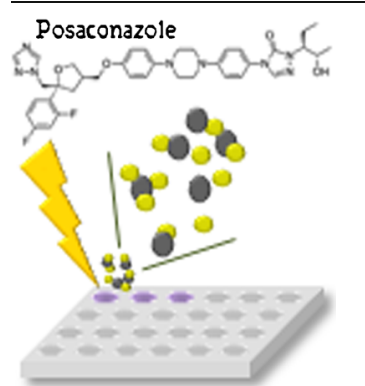

\begin{abstract}
A simple, rapid, and sensitive method for the detection of posaconazole using dispersive liquid-liquid microextraction (DLLME) coupled to surface-assisted laser desorption/ionization mass spectrometric detection (SALDI/MS) was developed. After the DLLME, posaconazole was detected using SALDI/MS with colloidal gold and a-cyano-4-hydroxycinnamic acid (CHCA) as the co-matrix. Under optimal extraction and detection conditions, the calibration curve, which ranged from 1.0 to $100.0 \mathrm{nM}$ for posaconazole, was observed to be linear. The limit of detection (LOD) at a signal-to-noise ratio of 3 was $0.3 \mathrm{nM}$ for posaconazole. This novel method was successfully applied to the determination of posaconazole in human urine samples.
\end{abstract} Key words: Surface-assisted laser desorption/ionization mass spectrometry, Dispersive liquid-liquid microextraction, Posaconazole, Urine sample

Received: 21 September 2014/Revised: 18 November 2014/Accepted: 3 December 2014/Published Online: 6 January 2015

\section{Introduction}

$\mathrm{P}$ osaconazole is a second-generation triazole fungicide that has become the most widely prescribed medication for treating invasive fungal infections $[1,2]$. Posaconazole is currently available as an oral suspension and shows high interindividual differences in oral bioavailability. The analytical methods used to monitor posaconazole have been reviewed by Ekiert et al. [3]. Different methods have been reported for analyzing posaconazole in human plasma [4-6]. Among these methods, LC/MS has become the most popular technique because of its sensitivity and specificity.

Despite the high sensitivity achieved by many analytical instruments, an extraction/preconcentration step is generally required for the determination of posaconazole in biological samples. Liquid-liquid extraction (LLE) [7] and solid-phase extraction (SPE) $[6,8]$ are the most common procedures for the extraction/preconcentration of posaconazole from plasma samples. Recently, dispersive liquid-liquid microextraction

Electronic supplementary material The online version of this article (doi:10.1007/s13361-014-1061-2) contains supplementary material, which is available to authorized users.

Correspondence to: Sarah Chang; e-mail: ychang@nknucc.nknu.edu.tw
(DLLME) has become a popular microextraction technique because of its rapidity, ease of operation, and low cost [9]. Despite the wide applicability of DLLME to drug analysis [10], DLLME has not yet been applied to the extraction of posaconazole.

SALDI/MS [11] has the advantages of high-throughput, ease of sample preparation, reduced background noise in the low-mass region, and better shot-to-shot reproducibility. Therefore, SALDI/MS is a practical tool for quantifying drugs in biological samples. However, the difficulties associated with establishing an analytical method for the determination of drug in biological samples primarily arise from the complexities of the sample matrices and the low analyte concentrations in the samples. For the rapid determination of drug in complex sample matrix, further attention should be paid to the development of a simple analytical procedure. The aim of this study was to develop a simple, rapid, and sensitive SALDI/MS method for the determination of posaconazole. To suppress the matrix effect and enhance sensitivity, DLLME was employed to extract posaconazole from an aqueous solution. Various parameters that affect the extraction efficiencies and analyte detection were investigated. The applicability of the method to the determination of posaconazole in human urine was also demonstrated. To our knowledge, this is the first report that demonstrates the use of SALDI/MS for the analysis of posaconazole. 


\section{Experimental}

\section{Chemicals and Solutions}

Posaconazole, CHCA, and carbon tetrachloride were purchased from Sigma (St. Louis, MO, USA). Dichloromethane and chlorobenzene were obtained from J.T. Baker (Phillipsburg, NJ, USA). Chloroform was obtained from Showa (Tokyo, Japan). Colloidal suspensions of palladium (10 ppm), gold (10 ppm), and silver $(10 \mathrm{ppm})$ were purchased from Purest Colloids (Westhampton, NJ, USA). Water purified with a Millipore Synergy water purification system (Billerica, MA, USA) was used for all solutions.

\section{DLLME Procedure}

The DLLME procedure has been described previously [12]. Forty microliters of the sediment phase was transferred to a separate sample vial using a $100 \mu \mathrm{L}$ HPLC syringe (Hamilton, Reno, NV, USA). The extract was evaporated to dryness. The extract residue was redissolved with $5 \mu \mathrm{L}$ of solution containing $80 \%$ methanol and $20 \%$ colloidal Au.

\section{SALDI/MS Measurements}

One microliter of the posaconazole solution was mixed with $1 \mu \mathrm{L}$ of colloidal Pd solution. Then, $1 \mu \mathrm{L}$ of the mixture was deposited onto a stainless steel target and dried in the air at room temperature. For samples with DLLME, $1 \mu \mathrm{L}$ of the extract solution was deposited onto a stainless steel target and dried at room temperature. Then, $1 \mu \mathrm{L}$ of the CHCA solution was applied on top of the colloidal Au prior to SALDI/MS analysis.

Mass spectrometry experiments were performed in positiveion mode on a reflectron-type time-of-flight mass spectrometer (Microflex, Bruker Daltonics, Bremen, Germany) with a flight length of $1.96 \mathrm{~m}$. The samples were irradiated with a $337 \mathrm{~nm}$ nitrogen laser at $20 \mathrm{~Hz}$. The generated ions were accelerated at a voltage of $19 \mathrm{kV}$. To obtain good signal-to-noise ratios, the laser energy settings were adjusted to slightly exceed the threshold, and each spectrum was acquired from an average of 100 laser pulses.

\section{Results and Discussion}

\section{Detection of Posaconazole by SALDI/MS}

In SALDI/MS, Au is the most commonly used SALDI matrix. Previously, Au NPs were found to be efficient SALDI matrices for the ionization of peptides [11], aminothiols [13], glutathiones [14], and small carbohydrates [15]. We investigated the use of colloidal $\mathrm{Au}, \mathrm{Ag}$, and Pd as SALDI matrices for the detection of posaconazole. The ion signals at $\mathrm{m} / \mathrm{z} 723.73$ corresponding to the sodium adduct ion of posaconazole were obtained for these three matrices. Figure 1a shows the mass spectrum of posaconazole using colloidal Au as SALDI matrices. These alkali ion adducts were commonly observed when using nanomaterials as SALDI matrices. When using colloidal Au as the SALDI matrix, the signal-to-noise $(\mathrm{S} / \mathrm{N})$ ratio of posaconazole was 4.0 and 2.2 times greater than the signal obtained using colloidal $\mathrm{Ag}$ and $\mathrm{Pd}$ as the SALDI matrices, respectively. To promote the ionization efficiency of posaconazole, a CHCA solution, a common organic matrix for MALDI/MS, was applied on top of the colloidal Au prior to SALDI/MS analysis. As shown in Figure 1b, the ion signal observed at $\mathrm{m} / \mathrm{z} 701.87$ was assigned to [posaconazole + $\mathrm{H}]^{+}$, respectively. Although some fragment ions of posaconazole were observed, the signal intensity of posaconazole was greatly increased because of the addition of CHCA. Posaconazole molecules were protonated via ion-molecule reactions with CHCA. In addition, posaconazole exhibited high affinity toward proton ions. The $\mathrm{S} / \mathrm{N}$ ratio of [posaconazole $+\mathrm{H}]^{+}$when using $\mathrm{CHCA}$ and colloidal $\mathrm{Au}$ as a co-matrix was 13.9 times higher than the $\mathrm{S} / \mathrm{N}$ ratio of [posaconazole $+\mathrm{Na}]^{+}$obtained when colloidal $\mathrm{Au}$ was used alone as the matrix. Hence, CHCA and colloidal Au were used as a co-matrix for all subsequent experiments.

\section{Optimization of DLLME}

To optimize the extraction efficiency by using DLLME, the amount of extracted posaconazole was monitored based on the ion intensities of [posaconazole $+\mathrm{H}]^{+}$in the three samples. The selection of the disperser solvent and the extraction solvent is a critical factor in DLLME. All combinations of the extraction solvents dichloromethane, carbon tetrachloride, chlorobenzene, and chloroform with acetonitrile, tetrahydrofuran, methanol, and acetone as disperser solvents were investigated. Fifty microliters of the extraction solvent was dissolved in $260 \mu \mathrm{L}$ of the disperser solvent. The combination of chloroform and acetone provided the highest extraction efficiency. The effect of the disperser solvent volume on the signal intensity was investigated from 200 to $270 \mu \mathrm{L}$. The results showed that the signal intensities increased when the disperser solvent volume increased from 200 to $260 \mu \mathrm{L}$. At lower volumes of acetone, the cloudy solution did not form as efficiently, resulting in decreased signal intensities. At higher volumes of acetone, the solubility of the posaconazole in water increased and the signal intensities decreased. To investigate the effect of the extraction solvent volume, several volumes of chloroform were subjected to the same DLLME procedures. From those samples, volumes of 20, 30, 40, 50, and $60 \mu \mathrm{L}$ of the sediment phase were collected for analysis from chloroform solutions of $30,40,50,60$, and $70 \mu \mathrm{L}$, respectively. The results showed that the signal intensities increased as the extraction solvent volume increased from 30 to $50 \mu \mathrm{L}$. At higher volumes of chloroform, the signal intensities decreased, which might be attributed to the formation of larger chloroform droplets. Hence, $50 \mu \mathrm{L}$ of the extraction solvent was used for all subsequent experiments.

Posaconazole is weakly basic because of the presence of heterocyclic nitrogen atoms $[3,16]$. The effect of $\mathrm{pH}$ on the signal intensities of posaconazole was investigated from 3.0 to 8.0 (see Figure S1 in Supplemental Material). The signal intensities of posaconazole increased as the $\mathrm{pH}$ of the solution was increased from 3.0 to 7.0 and then remained constant at $\mathrm{pH}$ values between 7.0 and 8.0. The $\mathrm{pK}_{\mathrm{a}}$ value of posaconazole $\mathrm{H}^{+}$ 


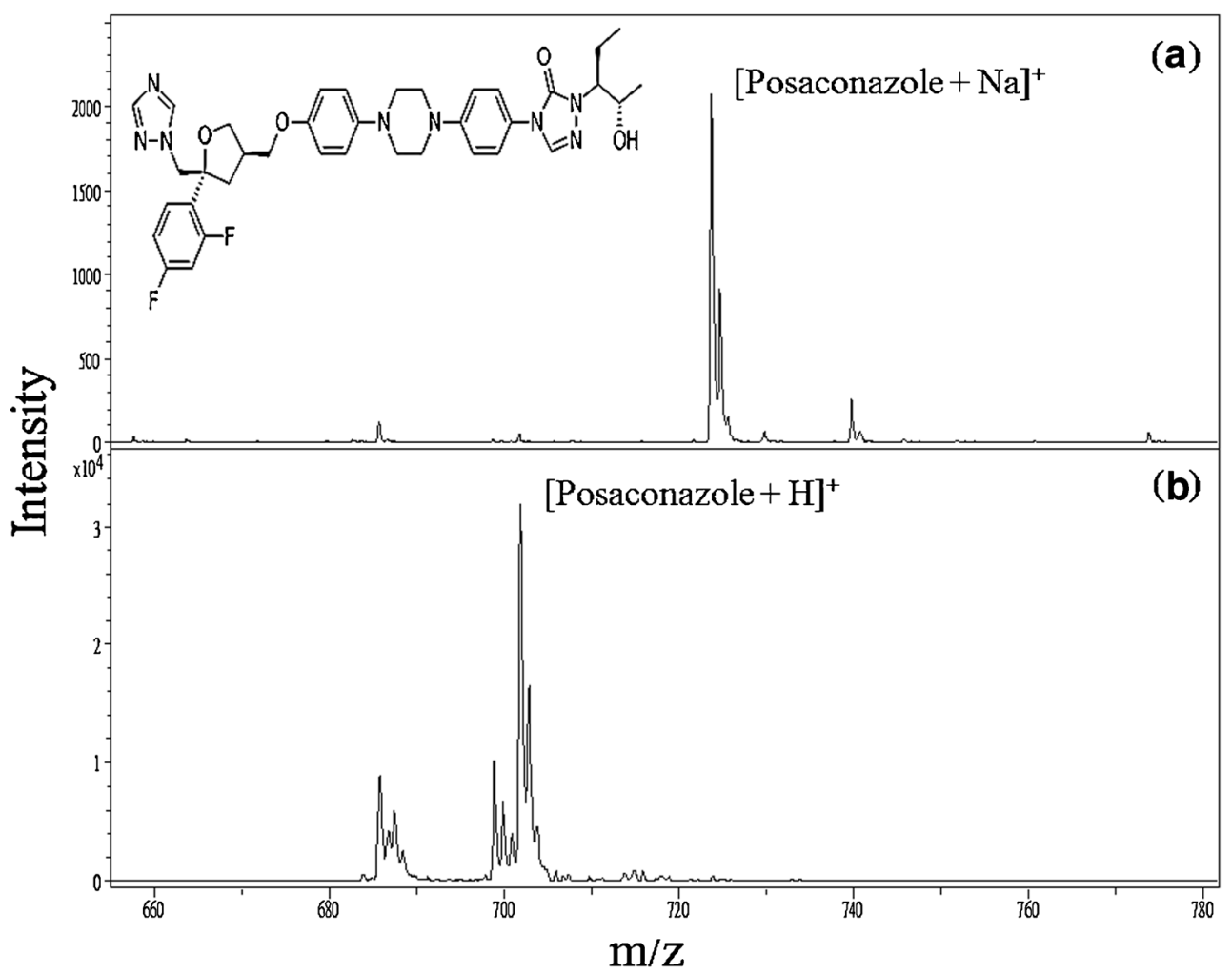

Figure 1. Mass spectra of posaconazole with (a) colloidal Au, (b) colloidal Au and CHCA matrices. The ions at $m / z=723.73$ and 701.87 correspond to [posaconazole $+\mathrm{Na}^{+}$and [posaconazole $\left.+\mathrm{H}\right]^{+}$, respectively. The concentration of the posaconazole was $10 \mu \mathrm{M}$

is 3.6. When the $\mathrm{pH}$ of the solution was greater than the $\mathrm{pK}_{\mathrm{a}}$ value of posaconazole $\mathrm{H}^{+}$, posaconazole existed in its neutral form and was efficiently extracted using the DLLME method. Therefore, the $\mathrm{pH}$ of the solution was set to 7 . The optimal extraction time was investigated within the range of 1 to $15 \mathrm{~min}$, while holding all other parameters constant. As expected, the observed signal intensities of posaconazole gradually increased as the extraction times increased from 1 to $3 \mathrm{~min}$, and reached a plateau after $3 \mathrm{~min}$. Thus, the extraction time was set to $3 \mathrm{~min}$. Owing to the large amount of surface contact between the solvent droplets and the aqueous sample, the mass transfer from the sample solution to the extraction solvent is very rapid. This rapid mass transfer is the most important advantage conferred by the DLLME technique.

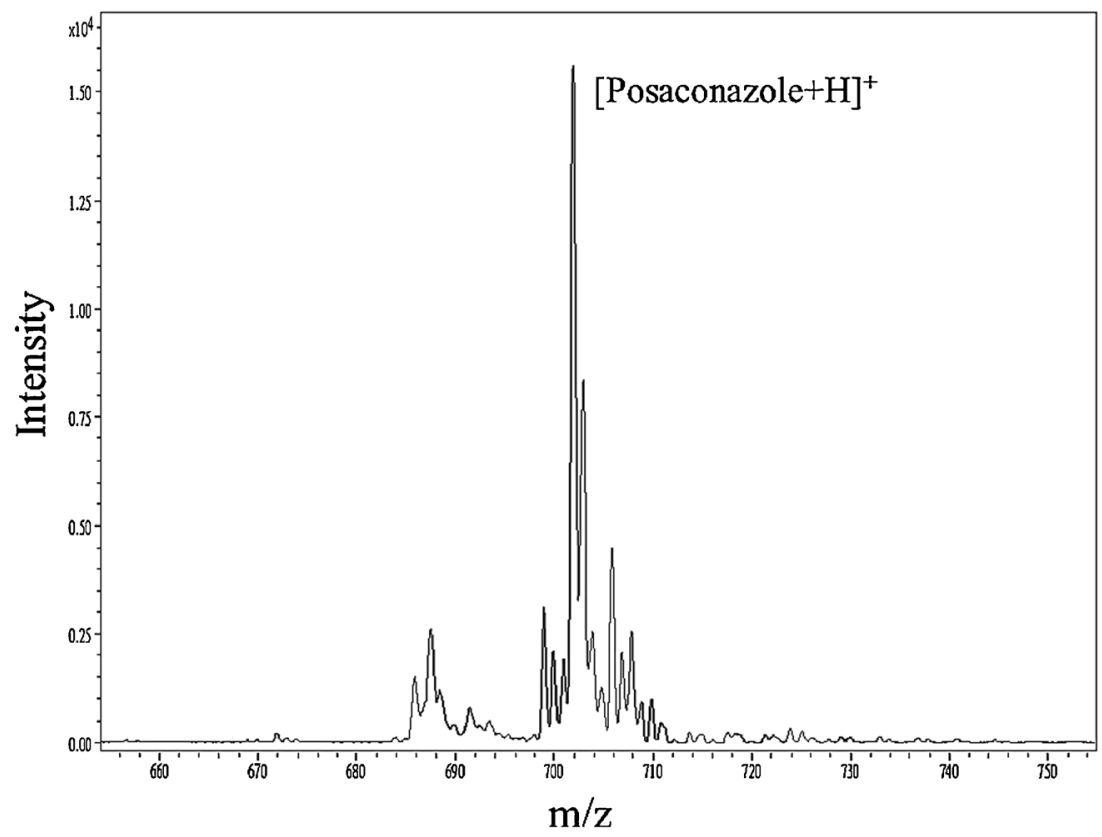

Figure 2. Mass spectrum of human urine sample obtained after extraction by DLLME 


\section{Analytical Characteristics}

A calibration curve for posaconazole was constructed over the concentration range $1.0-100.0 \mathrm{nM}$. A plot of the signal intensity of [posaconazole $+\mathrm{H}]^{+}$versus concentration presented good linearity $(\mathrm{y}=323077.0 \mathrm{x}+3.1)$ with a correlation coefficient (r) of $0.9982(n=8)$. The LOD and limit of quantitation (LOQ, $\mathrm{S} / \mathrm{N}=10$ ) values for posaconazole in aqueous solution were calculated to be 0.3 and $1.0 \mathrm{nM}$, respectively. The enrichment factor $(\mathrm{EF})$ was estimated by following a previously reported method [17]. With a sample-to-extract volume ratio of 200, the EF for posaconazole was calculated to be $1.11 \times 10^{2}$.

\section{Applications}

To evaluate the applicability of this method for clinical analyses, human urine spiked with posaconazole was used as a test sample. Without DLLME, a weak detected posaconazole ion was present when using direct SALDI/MS analysis. After treatment using the DLLME method, the posaconazole signal was greatly increased, as shown in Figure 2. The ions at $\mathrm{m} / \mathrm{z} 701.87$ correspond to [posaconazole $+\mathrm{H}]^{+}$. A calibration curve was constructed using the posaconazole-spiked urine in the concentration range $1.5-150.0 \mathrm{nM}$. The plot of the posaconazole signal intensity versus concentration exhibited good linearity $(\mathrm{y}=169403.3 \mathrm{x}+4.0)$ with a correlation coefficient $(\mathrm{r})$ of $0.9983(n=8)$. The LOD and LOQ values for posaconazole in urine were calculated to be 0.4 and $1.5 \mathrm{nM}$, respectively. The LOD obtained with this developed method was lower than that reported using capillary electrophoresis (CE) coupled with SPE and sample stacking technique [6]. In comparison to LC-MS/MS coupled with SPE, this developed method has similar sensitivity but simpler sample preparation procedure [18].

The within-day and between-day effects on the accuracy and precision of the method were evaluated using urine samples that were spiked with low $(1.5 \mathrm{nM})$, medium $(15 \mathrm{nM})$, and high (150 $\mathrm{nM}$ ) concentrations of posaconazole. The within-day and between-day accuracies for the three spiked levels of posaconazole in urine, which are represented as relative error (RE), were between $-2.6 \%$ and $-1.8 \%$, and between $4.8 \%$ and $5.9 \%$, respectively. The within-day and between-day precisions for the three spiked levels of posaconazole, where precisions are represented as RSDs, were $5.0 \%-8.8 \%$ and $6.6 \%-10.6 \%$, respectively. These obtained values meet the accuracy and precision criteria set forth in the guidelines on bioanalytical method validation [19]. Combining the rapidity of SALDI/MS and the simplicity of DLLME, the analysis of posaconazole in urine samples can be readily performed in less than $10 \mathrm{~min}$. This newly developed SALDI/MS method is suitable for the clinical screening of posaconazole in human urine samples.

\section{Acknowledgments}

The authors acknowledge financial support for this work by the National Science Council (NSC 102-2113-M-017-001-) of Taiwan.

\section{References}

1. Munayyer, H.K., Mann, P.A., Chau, A.S., Yarosh-Tomaine, T., Greene, J.R., Hare, R.S., Heimark, L., Palermo, R.E., Loebenberg, D., McNicholas, P.M.: Posaconazole is a potent inhibitor of sterol $14 \alpha$-demethylation in yeasts and molds. Antimicrob. Agents Chemother. 48, 3690-3696 (2004)

2. Pitisuttithum, P., Negroni, R., Graybill, J.R., Bustamante, B., Pappas, P., Chapman, S., Hare, R.S., Hardalo, C.J.: Activity of posaconazole in the treatment of central nervous system fungal infections. J. Antimicrob. Chemother. 56, 745-755 (2005)

3. Ekiert, R.J., Krzek, J., Talik, P.: Chromatographic and electrophoretic techniques used in the analysis of triazole antifungal agents-a review. Talanta 82, 1090-1100 (2010)

4. Kahle, K., Langmann, P., Schirmer, D., Lenker, U., Keller, D., Helle, A., Klinker, H., Heinz, W.J.: Simultaneous Determination of voriconazole and posaconazole concentrations in human plasma by high-performance liquid chromatography. Antimicrob. Agents Chemother. 53, 3140-3142 (2009)

5. Shen, J.X., Krishna, G., Hayes, R.N.: A sensitive liquid chromatography and mass spectrometry method for the determination of posaconazole in human plasma. J. Pharm. Biomed. Anal. 43, 228-236 (2007)

6. Liao, H.W., Lin, S.W., Wu, U.I., Kuo, C.H.: Rapid and sensitive determination of posaconazole in patient plasma by capillary electrophoresis with field-amplified sample stacking. J. Chromatogr. A. 1226, 48-54 (2012)

7. Chhun, S., Rey, E., Tran, A., Lortholary, O., Pons, G., Jullien, V.: Simultaneous quantification of voriconazole and posaconazole in human plasma by high-performance liquid chromatography with ultra-violet detection. J. Chromatogr. B. 852, 223-228 (2007)

8. Cunliffe, J.M., Noren, C.F., Hayes, R.N., Clement, R.P., Shen, J.X.: A high-throughput LC-MS/MS method for the quantitation of posaconazole in human plasma: implementing fused core silica liquid chromatography. J. Pharm. Biomed. Anal. 50, 46-52 (2009)

9. Berijani, S., Assadi, Y., Anbia, M., Hosseini, M.R.M., Aghaee, E.: Dispersive liquid-liquid microextraction combined with gas chromatographyflame photometric detection very simple, rapid and sensitive method for the determination of organophosphorus pesticides in water. J. Chromatogr. A. 1123, 1-9 (2006)

10. Yazdi, A.S., Razavi, N., Yazdinejad, S.R.: Separation and determination of amitriptyline and nortriptyline by dispersive liquid-liquid microextraction combined with gas chromatography flame ionization detection. Talanta $\mathbf{7 5}$, 1293-1299 (2008)

11. McLean, J.A., Stumpo, K.A., Russell, D.H.: Size-selected (2010 nm) gold nanoparticles for matrix assisted laser desorption ionization of peptides. J. Am. Chem. Soc. 127, 5304-5305 (2005)

12. Cheng, M.C., Chi, K.M., Chang, S.Y.: Detection of digoxin in urine samples by surface-assisted laser desorption/ionization mass spectrometry with dispersive liquid-liquid microextraction. Talanta 115, 123-128 (2013)

13. Huang, Y.F., Chang, H.T.: Nile red-adsorbed gold nanoparticle matrixes for determining aminothiols through surface-assisted laser desorption/ionization mass spectrometry. Anal. Chem. 78, 14851493 (2006)

14. Huang, Y.F., Chang, H.T.: Analysis of adenosine triphosphate and glutathione through gold nanoparticles assisted laser desorption/ionization mass spectrometry. Anal. Chem. 79, 4852-4859 (2007)

15. Su, C.L., Tseng, W.L.: Gold nanoparticles as assisted matrix for determining neutral small carbohydrates through laser desorption/ ionization time-of-flight mass spectrometry. Anal. Chem. 79, 16261633 (2007)

16. Yahaya, N., Sanagi, M.M., Nur, H., Ibrahim, W.A.W., Kamaruzaman, S., Aboul-Enein, H.Y.: Solid-phase membrane tip extraction combined with liquid chromatography for the determination of azole antifungal drugs in human plasma. Anal. Methods. 6, 3375-3381 (2014)

17. Hsu, C.C., Whang, C.W.: Microscale solid phase extraction of glyphosate and aminomethylphosphonic acid in water and guava fruit extract using alumina-coated iron oxide nanoparticles followed by capillary electrophoresis and electrochemiluminescence detection. J. Chromatogr. A. 1216, $8575-8580$ (2009)

18. Beste, K.Y., Burkhardt, O., Kaever, V.: Rapid HPLC-MS/MS method for simultaneous quantitation of four routinely administered triazole antifungals in human plasma. Clin. Chim. Acta. 413, 240-245 (2012)

19. Guidance for industry: bioanalytical method validation, US Department of Health and Human Services, Food and Drug Administration, Center for Drug Evaluation and Research, Center for Veterinary Medicine, Rockville, MD, USA. http://www.fda.gov/cder/guidance/index.htm (2001) 